\title{
The effects of smoking on the expression of gelatinases in chronic periodontitis: a cross-sectional study
}

\section{Rahşan Atasoy ŞENTÜRK(a) \\ Yasemin SEZGiN ${ }^{(b)}$ \\ Sule BULUT(b) \\ Binnaz Handan ÖZDEMIR(c)}

(a)Koru Hospital, Ankara, Turkey.

(b)Baskent University, Faculty of Dentistry, Department of Periodontology, Bahçelievler, Ankara, Turkey.

(c)Baskent University, Faculty of Medicine, Department of Pathology, Bahçelievler, Ankara, Turkey.

Declaration of Interest: The authors certify that they have no commercial or associative interest that represents a conflict of interest in connection with the manuscript.

\section{Corresponding Author:}

YaseminSezgin, Ph.D., DDS

E-mail: yasemin_tocak@hotmail.com

hitps://doi.org/10.1590/1807-3107bor-2018.vol32.0114

Submitted: March 18, 2018

Accepted for publication: August 21, 2018

Last revision: September 17, 2018
Abstract: Smokers have a risk of developing periodontal disease. Matrix metalloproteinases (MMP) play a significant role in periodontal tissue destruction. In this study possible relationship between smoking and gingival tissue expression of gelatinases in chronic periodontitis patients relative to periodontally healthy subjects was investigated. Forty chronic periodontitis patients $(20$ smokers and 20 non-smokers) and forty periodontally healthy subjects (20 smokers and 20 non-smokers) were enrolled. The clinical periodontal measurements recorded, and gingival tissues harvested after that. After histologic evaluation, matrix metalloproteinases -2 and -9 expressions were analyzed immunohistochemically. In nonsmokers, higher expression of metalloproteinases -2 and -9 detected in chronic periodontitis group compared to the periodontally healthy group. In the smoker chronic periodontitis group, the expression of metalloproteinases- 2 was lower than nonsmoker chronic periodontitis group. Statistically significant differences detected between smoker and nonsmoker periodontally healthy groups in metalloproteinases-2 expression. For metalloproteinases-9 expression, smoker chronic periodontitis group has lower values than nonsmoker chronic periodontitis group. In periodontally healthy group smokers showed higher metalloproteinases -9 expressions than non- smokers. Present findings support the role of gelatinases in chronic periodontitis pathogenesis. Based on the current results we conclude that smoking alters the expression of gelatinases in gingival tissues.

Keywords: Gelatinases; Matrix Metalloproteinases; Periodontitis; Smoking.

\section{Introduction}

Periodontitis is defined as an infectious-inflammatory disease that occurs by the degradation of supporting tissues of the teeth. ${ }^{1}$ Although primer etiologic factor of periodontitis is pathogenic bacterial species, risk factors play a critical role by changing the susceptibility or resistance of individuals to the disease. Risk factors include lifestyle (e.g., smoking and alcohol consumption), medical conditions (e.g., diabetes mellitus, obesity, osteopenia), age, gender. ${ }^{2}$ Among these risk factors; smoking is the most potent modifiable risk factor second to bacterial plaque. ${ }^{3}$ Experimental evidence has shown that cigarette smoking increases the 
susceptibility, severity, and progression of periodontal diseases. Furthermore, smoking has adverse effects on healing outcomes following surgical and nonsurgical periodontal therapies. ${ }^{4,5,6,7}$

Due to obtaining different treatment outcomes, prevalence and severity rates for smokers and nonsmokers, researchers paid their attention to understand the mechanism by which smoking interferes with periodontal disease activity.

Matrix metalloproteinases (MMPs) are involved not only in physiological development and tissue remodeling but also in pathological tissue degradation. This group of 23 enzymes divided into five major subgroups based on substrate specificity and molecular structure. These groups are named as collagenases (MMP-1,-8,-13), gelatinases (MMP-2, MMP-9), stromelysins (MMP-3, -10,-11),membrane-type MMPs (MMP-14, -15, -16, -17) and others. Increased levels of MMPs are essential for the destruction of periodontal tissues. ${ }^{8,9}$

In previous studies tumor necrosis factor (TNF) $-\alpha$, which plays a role in the transcription levels of MMP genes, has been detected in increased levels in smokers compared to non-smokers. ${ }^{10}$ Also, higher elastase activity levels and its correlation with MMP activity reported for smokers compared with non-smokers controls. ${ }^{11}$ The effects of smoking on increased MMP levels has been suggested as one possible reason for the increased risk and/or progression in periodontitis, but the exact mechanism by which smoking implements its harmful effects on periodontium is still remained unknown. ${ }^{11}$

MMP-2 and MMP-9, called gelatinases, are one of the major MMPs that plays important roles in periodontitis. To date, several studies showed the increased levels of gelatinases in periodontal disease and only a limited number of the study investigated the effects of smoking on gelatinases. ${ }^{12,13,14}$ However, to the authors' knowledge, no previous study has directly investigated the possible relationship between smoking and gingival tissue gelatinases expression (MMP-2 and MMP-9) in chronic periodontitis patients relative to periodontally healthy subjects.

Therefore this study is conducted to comparatively evaluate the expression of MMP-2 and MMP-9 in smoker vs. non-smoker chronic periodontitis patients as well as periodontally healthy subjects. We hypothesized that smoking might interfere with periodontal disease by effecting MMP-2 and MMP-9 expression.

\section{Methodology}

\section{Study group population}

A total of 80 subjects ( 35 females, 45 males, with an age range of 16-62 years) included in this study from those patients seeking dental treatment at the Baskent University School of Dentistry between February 2010 and December 2012. The subjects included after receiving a detailed description of the procedures and having signed informed consent. The study protocol approved by Baskent University Institutional Review Board and Ethics Committee (D-KA11/07). This cross-sectional clinical trial, which examines the data at one particular time point, was registered at ClinicalTrials.gov (NCT03426241) and conducted by the Helsinki Declaration of 1975, as revised in 2000. This study reported by the Analysis Strengthening the Reporting of Observational Studies in Epidemiology (STROBE) Statement. ${ }^{15}$

The dental, as well as medical histories, were obtained from the patient. Systemically healthy patients who had not received any antibiotic therapy and periodontal therapy in the last six months and have a minimum of 20 teeth (excluding third molars) included in the study. Exclusion criteria included the following: patients with systemic disease; pregnant and/or lactating women; patients taking any medications that may influence the research. Of the 93-screened patients, 80 eligible and consented subjects were classified into four groups as follows: Group 1: 20 smoker chronic periodontitis (S-CP); Group 2: 20 non-smoker chronic periodontitis (NS-CP); Group 3: 20 periodontally healthy smoker subjects (S-H); and

Group 4: 20 periodontally healthy non-smoker subjects (NS-H)

First, those who claimed to have never smoked were recruited into the non-smoker groups, while subjects who reported smoking $\geq 10$ cigarettes/day for more than five years drafted into the smoker groups. ${ }^{16}$ Chronic periodontitis $(\mathrm{CP})$ patients diagnosed by the clinical criteria stated in the consensus report of the 
World Workshop in Periodontitis. ${ }^{17}$ All patients had at least 20 teeth with four teeth in each jaw with probing depth (PD) of $\geq 5 \mathrm{~mm}$, clinical attachment level (CAL) of $\geq 4 \mathrm{~mm}$, and $\geq 50 \%$ alveolar bone loss in at least two quadrants. Evaluation of the extent and severity of alveolar bone loss performed radiographically. Patients with no sites with probing depth $\geq 3$, attachment loss, GI $<0.5^{18,19}$ or radiographic evidence of bone loss recruited into the periodontally healthy group.

\section{Clinical measurements}

The clinical periodontal parameters, including $\mathrm{PD}, \mathrm{CAL}$, plaque index $(\mathrm{PI}){ }^{20}$ gingival index $(\mathrm{GI})^{18}$ were recorded at six sites on each tooth present, except the third molars using a periodontal probe (Nordent Manufacturing Inc., USA). All clinical examinations were carried out by a single calibrated examiner (RAS)

\section{Intra-examiner Calibration}

Before performing clinical recordings, the examiners evaluated clinical measurements from five patients twice, 48 hours apart. Calibration was accepted if $\geq 90$ of the recordings could reproduce within a 1-mm difference.

\section{Gingival tissue sampling}

In chronic periodontitis group, after recording the clinical parameters, gingival tissues were harvested from the deepest periodontal pocket with the help of Gracey's curettes. Whereas in the periodontally healthy group, gingival tissues collected during crownlengthening surgery or extraction of teeth for orthodontic reasons. In both groups, the samples include both sulcus/ pocket epithelium and underlying connective tissues. (approximately $2 \mathrm{~mm}$ wide $\mathrm{X} 2 \mathrm{~mm}$ high $\mathrm{X} 1$ to $3 \mathrm{~mm}$ thick)

\section{Pathologic analyses}

\section{Histologic evaluation}

All biopsies were fixed in $10 \%$ formalin and then embedded in paraffin blocks. For each sample, several $4 \mu \mathrm{m}$ serial sections obtained and stained with hematoxylin and eosin. Tissue sections examined using the light microscopy at a magnification of 200X. (Olympus BX53,
Tokyo, Japan) Inflammatory cells and fibroblast proliferation scored according to the following scale: 1: no inflammatory cells/ fibroblast infiltration, 2: inflammatory cells/fibroblast infiltration covering less than $30 \%$ of the $0.25 \mathrm{~mm}^{2}$ area, 3: inflammatory cells/fibroblast infiltration covering more than $30 \%$ of the $0.25 \mathrm{~mm}^{2}$ area. Neovascularization was scored semiquantitatively as following: $1: \leq 10$ microvessels in all areas, 2: 10-30 microvessels in all areas, $3: \geq 30$ microvessels in all areas.

\section{Immunohistochemical analysis of MMP-2 and MMP-9}

For immunohistochemical examination, three $\mu \mathrm{m}$-thick sections were deparaffinized and mounted on ploy-L-lysine coated slides. The tissue sections in citrate buffer were heated in a microwave oven for 15 minutes at maximum power $(700 \mathrm{~W})$. After that, the tissue sections cooled at room temperature for 20 minutes. Tissue sections were then incubated with MMP-2 antibodies for 2 hours in a humidified chamber at room temperature. After washing in buffer, the slides were incubated with biotinylated goat anti-polyvalent for 15 minutes and in streptavidin peroxidase for 15 minutes at room temperature. The tissue slides then developed for 12 minutes in diaminobenzidine. The slides subsequently counterstained with hematoxylin. Same procedures repeated with MMP-9 antibodies with a 2 hour incubation period. MMP-2 and MMP-9 evaluated semiquantitatively according to presence or absence of the expression as follows: MMP-2 (-): No staining, MMP-2 (+): positive staining, MMP-9 (-): No staining, MMP-9 (+): positive staining.

\section{Statistical analysis}

The sample size estimation was performed based on previous studies. ${ }^{21,22}$ A sample size of 20 participants for each group was required to detect at least $\% 40$ difference between the groups with a power of $80 \%$ and $\alpha=0.05$. The sample size was calculated using OpenEpi (Open Source Epidemiologic Statistics for Public Health) version 3.5.4. ${ }^{23}$ Since there are four study groups in the present study and a need of 20 participants in each group, eighty subjects included in this study. The distribution of continuous variables determined by the Kolmogorov-Smirnov test. The 
Levene test used for the evaluation of homogeneity of variances. While the nonparametric test statistics evaluated the quantitative variables for which the assumptions on above not met (Inflammatory cells, fibroblast proliferation, neovascularization), otherwise, the parametrical test statistics were applied for data (Plaque index, gingival index, probing depth, clinical attachment level) analyses. The homogeneity of the age among the groups tested with One-way ANOVA, and statistically significant differences were detected. Thus by treating age as a covariant, two way independent ANCOVA was used to compare the differences in clinical parameters between the study groups (S-CP, NS-CP, S-H, NS-H). Histologic parameters compared between the study groups by Kruskal Wallis variance analysis (S-CP, NS-CP, S-H, NS-H). Where there were significant differences Post hoc test was used to determine which groups significantly differ. The chi-squared test used to detect differences in the frequencies of gender, MMP-2, MMP-9 among the groups. The Spearman correlation coefficient observed the possible correlations between the clinical periodontal measurements and pathologic variables. Statistical examination performed using the SPSS version 15.0 statistical software package.

\section{Results}

\section{Clinical analyses}

Demographic variables and mean values of clinical measurements outlined in Table 1. Statistically, significant differences found between groups in regards to the mean age. Non-smoker periodontally healthy group was younger than the non-smoker and smoker $\mathrm{CP}$ groups $(\mathrm{p}<0.05)$. Smoker periodontally healthy group is more youthful than smoker CP $(\mathrm{p}<0.05)$. Pearson's Chi-Square test examined the distribution of sex ratios and no differences detected among the study groups.

The periodontally healthy group exhibited significantly lower values in all clinical periodontal measurements than the $\mathrm{CP}$ group $(\mathrm{p}<0.05)$. In both $\mathrm{CP}$ and periodontally healthy groups, the PI, $\mathrm{PD}, \mathrm{CAL}$ values were similar in smokers to that of non-smokers $(p>0.05)$. In periodontally healthy group GI values were identical in both smokers and non-smokers whereas smokers in CP group exhibited lower GI values than non-smoker CP group $(\mathrm{p}=0.001)$.

\section{Pathologic examination}

\section{Histologic evaluation}

The results of histologic assessments outlined in Table 2. In smoker CP group inflammation, vascularization and fibroblastic proliferation scores are lower than the non-smoker $\mathrm{CP}$ group $(\mathrm{p}<0.01$, $\mathrm{p}$ $<0.05, p<0.01$ respectively). In contrast, there was no statistically significant differences between smokers and non-smokers periodontally healthy group regarding inflammation, neovascularization and fibroblastic proliferation scores $(p>0.05)$. When a comparison made between $\mathrm{CP}$ and periodontally healthy groups; $\mathrm{CP}$ group revealed higher inflammation, vascularization and fibroblastic proliferation scores in both smokers and non-smokers subgroups compared to those of the periodontal healthy smoker and non-smoker subgroups $(\mathrm{p}<0.05)$ (Figures 1 and 2).

Table 1. Clinical measurements (mean \pm standart deviations) of smoker and non-smoker chronic periodontitis and periodontally healthy subjects.

\begin{tabular}{|c|c|c|c|c|}
\hline \multirow{3}{*}{ Clinical variable } & \multicolumn{2}{|c|}{ Chronic periodontitis } & \multicolumn{2}{|c|}{ Periodontally healthy $n=40$} \\
\hline & Smokers & Non-smokers & Smokers & Non-smokers \\
\hline & $n=20$ & $n=20$ & $n=20$ & $\mathrm{n}=20$ \\
\hline Age(years) & $47.90 \pm 7.56$ & $46.50 \pm 10.95$ & $38.30 \pm 10.89 b$ & $33.70 \pm 13.52^{a, b}$ \\
\hline Male: female & $12: 08$ & 13:07 & $12: 08$ & $08: 12$ \\
\hline Probing Depth (mm) & $4.23 \pm 0.91$ & $4.75 \pm 0.90$ & $2.26 \pm 0.27$ & $2.17 \pm 0.21$ \\
\hline Clinical attachment level (mm) & $4.77 \pm 1.24$ & $5.51 \pm 1.47$ & $2.31 \pm 0.30$ & $2.21 \pm 0.23$ \\
\hline Plaque Index (PI) & $1.26 \pm 0.38$ & $1.34 \pm 0.41$ & $0.94 \pm 0.20$ & $0.86 \pm 0.19$ \\
\hline Gingival Index (GI) & $1.36 \pm 0.35^{a}$ & $1.79 \pm 0.26$ & $0.90 \pm 0.29$ & $1.04 \pm 0.24$ \\
\hline
\end{tabular}

aSignificantly lower than the non-smoker chronic periodontitis group; 'Significantly lower than the smoker chronic periodontitis group. 
Table 2. Histologic measurements (mean \pm standart deviations) of smoker and non-smoker chronic periodontitis and periodontally healthy subjects.

\begin{tabular}{lcccc}
\hline & \multicolumn{2}{c}{ Chronic periodontitis } & \multicolumn{2}{c}{ Periodontally healthy } \\
\cline { 2 - 5 } Histologic measurements & Smokers & Non-smokers & Smokers & Non-smokers \\
\cline { 2 - 5 } & $\mathrm{n}=20$ & $\mathrm{n}=20$ & $\mathrm{n}=20$ & $\mathrm{n}=20$ \\
\hline Inflammation & $2.10 \pm 0.85^{\mathrm{a}, \mathrm{b}}$ & $2.85 \pm 0.37^{\mathrm{b}}$ & $1.40 \pm 0.68$ & $1.25 \pm 0.44$ \\
Neovascularization & $2.40 \pm 0.60^{\mathrm{a}, \mathrm{b}}$ & $2.85 \pm 0.37^{\mathrm{b}}$ & $1.60 \pm 0.60$ & $1.60 \pm 0.69$ \\
Fibroblastic proliferation & $2.00 \pm 0.72^{\mathrm{a}, \mathrm{b}}$ & $2.85 \pm 0.37^{\mathrm{b}}$ & $1.50 \pm 0.69$ & $1.35 \pm 0.67$ \\
\hline
\end{tabular}

aSignificantly lower than the non-smoker chronic periodontitis group; 'Significantly higher than the periodontally healthy group.

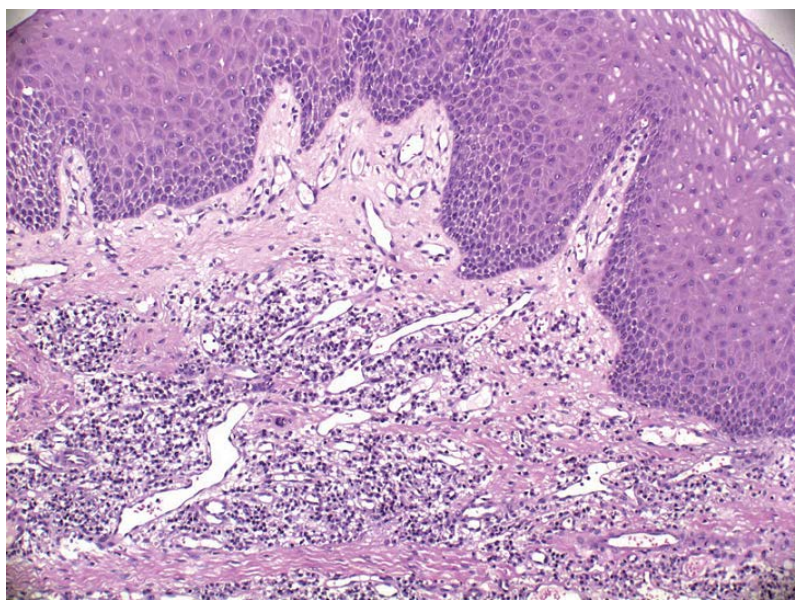

Figure 1. Photomicrographs of gingival specimens showing a large number of the diffuse lymphocyte-rich mononuclear cell infiltrate under the superficial epithelium, especially around vascular structures. Also, microvascular proliferation in the vicinity of inflammation is also clearly detected. (Hematoxylin and eosin $\mathrm{x} 200$ ) (CP group).

\section{Immunohistochemical analysis}

The immunohistochemical findings are shown in Table 3. In smoker CP group the expression of MMP-2 was lower than nonsmoker $\mathrm{CP}$ group $(\mathrm{p}<0.05)$. There were no statistically significant differences between smoker and non-smoker periodontally healthy groups in MMP-2 expression.

Concerning MMP-9 positivity, smoker CP group has lower values than the non-smoker $\mathrm{CP}$ group. In contrast, smoker periodontally healthy group showed higher MMP-9 positivity than non-smoker periodontally healthy group (Figure 3 and 4).

When a comparison made between chronic periodontitis and periodontally healthy groups, higher MMP-2 and MMP-9 positivity were detected in the periodontitis group $(\mathrm{p}<0.05)$.

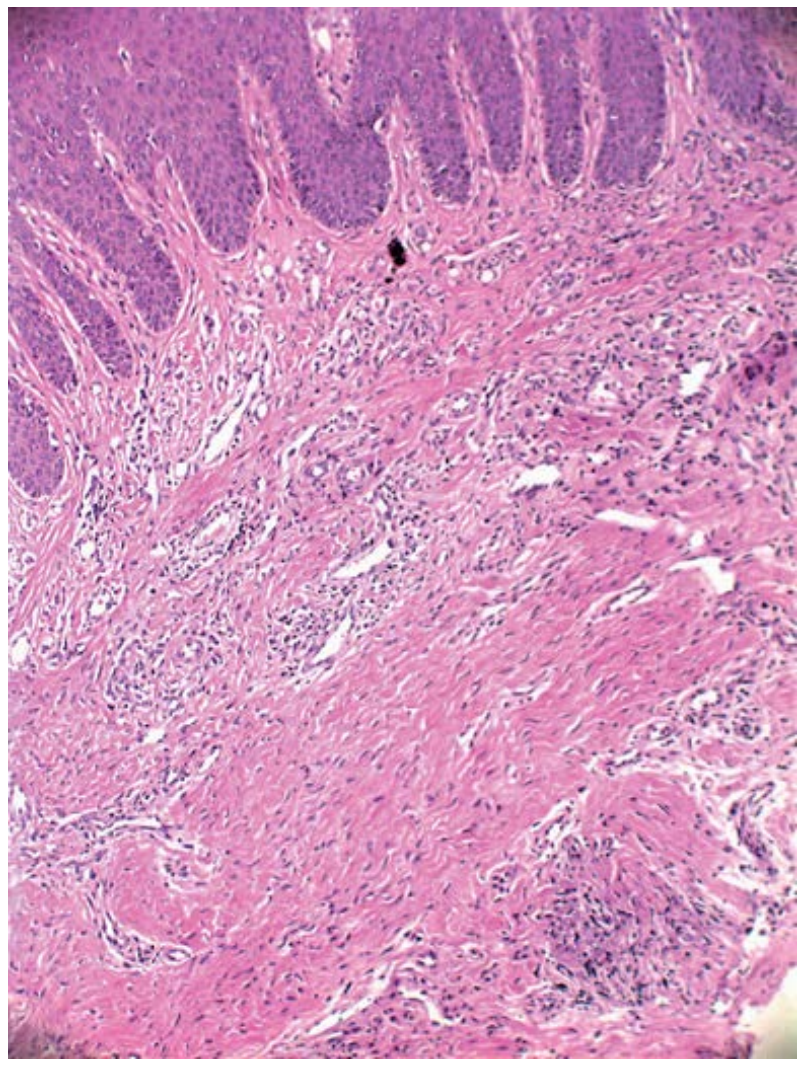

Figure 2. Photomicrographs of gingival specimens showing a small number of the focal mononuclear cell infiltrate under the superficial epithelium, especially around vascular structures. Although the inflammation is minimal, it is noteworthy that the microvascular proliferation in these areas is moderate. (Hematoxylin and eosin $\times 200$ ) (S-CP group).

Spearman correlation analyses revealed that in smoker CP group there are positive correlations between PI with inflammation and vascularization scores $(p=0.013, p=0.039$, respectively). Also GI positively correlated with inflammation, neovascularization and fibroblastic proliferation scores $(p=0.000, p=0.000, p=0.018$, respectively). 
Table 3. Distribution of immunohistochemical findings of smoker and non-smoker chronic periodontitis and periodontally healthy subjects.

\begin{tabular}{lcccc}
\hline & \multicolumn{2}{c}{ Chronic periodontitis } & \multicolumn{2}{c}{ Periodontally healthy } \\
\cline { 2 - 5 } Immunohistochemical findings & Smokers & Non-smokers & Smokers & Non-smokers \\
\cline { 2 - 5 } & $\mathrm{n}=20$ & $\mathrm{n}=20$ & $\mathrm{n}=20$ & $\mathrm{n}=20$ \\
\hline MMP-2 (+) & $12^{\mathrm{a}, \mathrm{c}}$ & $18^{\mathrm{c}}$ & 5 & 3 \\
MMP-2 (-) & 8 & 2 & 15 & 17 \\
MMP-9 (+) & $15^{\mathrm{a}, \mathrm{c}}$ & $20^{\mathrm{c}}$ & $10^{\mathrm{b}}$ & 3 \\
MMP-9 (-) & 5 & 0 & 10 & 17 \\
\hline
\end{tabular}

aSignificantly lower than the non-smoker chronic periodontitis group; 'Significantly higher than the non-smoker periodontally healthy group; cSignificantly higher than a periodontally healthy group.

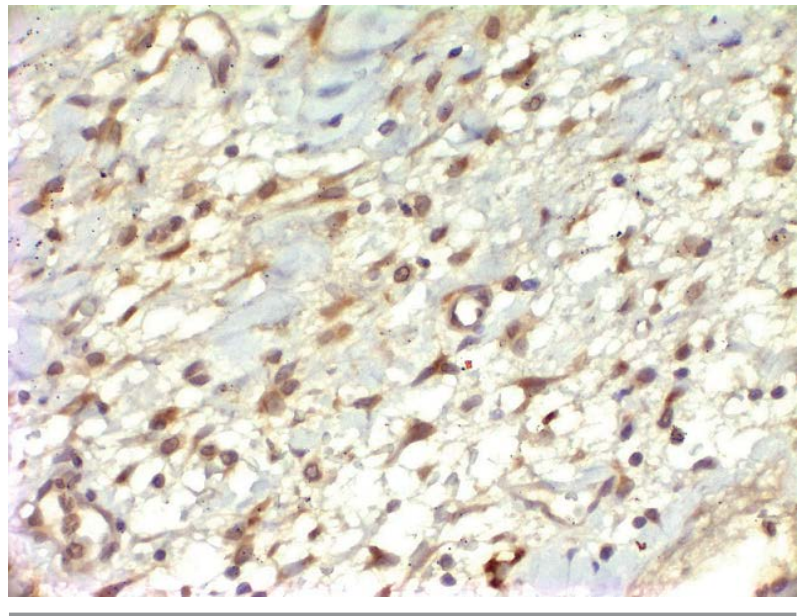

Figure 3. By immunostaining with antibodies anti-MMP-9, MMP-9 positive staining observed in fibroblasts which are showing moderate proliferation. There are quite a few negative staining inflammatory cells mixed with fibroblasts in between. (Immunostaining with antibodies anti-MMP-9, x400) (S-CP group).

PD and CAL positively correlated with inflammation and fibroblastic proliferation scores (for PD $\mathrm{p}=0.020$, $\mathrm{p}=0.011$; for CAL $\mathrm{p}=0.032, \mathrm{p}=0.032$, respectively). In smoker CP group, MMP-2 and MMP-9 positively correlated with inflammation, neovascularization and fibroblastic proliferation scores (for MMP- $2 p=0.038$, $\mathrm{p}=0.010, \mathrm{p}=0.008$; for MMP-9 $\mathrm{p}=0.003, \mathrm{p}=0.006$, $p=0.002$, respectively).

For non-smoker CP group, GI and MMP-2 positively correlated with inflammation, vascularization and fibroblastic proliferation scores (for GI $p=0.026$, $\mathrm{p}=0.026, \mathrm{p}=0.026$; for MMP-2 $\mathrm{p}=0.000, \mathrm{p}=0.000$, $\mathrm{p}=0.000$ respectively).

In smoker periodontally healthy group, PI, GI and MMP-9 positively correlated with inflammation,

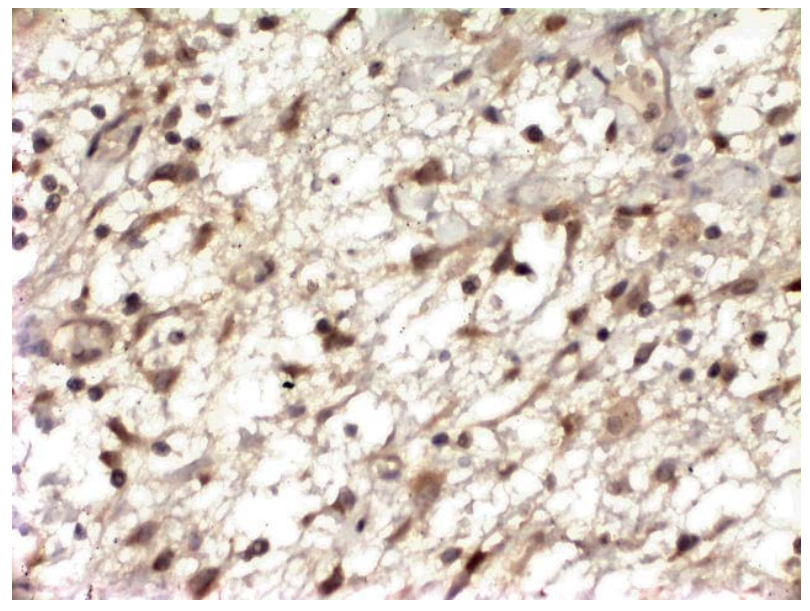

Figure 4. By immunostaining with antibodies anti-MMP-2, positive cytoplasmic staining with MMP-2 observed in a majority of fibroblasts. Inflammatory cells showing negative staining with mixed fibroblasts are noteworthy. (Immunostaining with antibodies anti-MMP-2, x400) (CP group).

vascularization and fibroblastic proliferation scores (for PI $p=0.018, p=0.012, p=0.045$; for GI $p=0.009$, $\mathrm{p}=0.001, \mathrm{p}=0.001$; for MMP-9 $\mathrm{p}=0.045, \mathrm{p}=0.001$, $\mathrm{p}=0.000$ respectively). MMP-2 possitively correlated with vascularization and fibroblastic proliferation scores in smoker periodontally healthy group ( $p=0.006, p=0.000$, respectively).

In non-smoker periodontally healthy group, GI, MMP-2 and MMP-9 positively correlated with vascularization and fibroblastic proliferation (for GI $\mathrm{p}=0.06, \mathrm{p}=0.041$; for MMP-2 $\mathrm{p}=0.001, \mathrm{p}=0.000$; for MMP-9 $p=0.001, p=0.000$, respectively); PD positively correlated with fibroblastic proliferation in non-smoker periodontally healthy group $(p=0.035)$. 


\section{Discussion}

In this cross-sectional study, we analyzed the gingival tissue expression of MMP-2 and MMP-9 in smoker vs. non-smoker chronic periodontitis patients as well as periodontally healthy subjects with the aim of explaining the possible mechanism by which smoking implements its harmful effects on periodontal tissues. The clinical periodontal measurements recorded and obtained gingival samples analyzed from 40 chronic periodontitis patients (20 smokers and 20 non-smokers) and 40 periodontally healthy subjects (20 smokers and 20 non-smokers). As previous studies reported the high susceptibility rates for development and progression of periodontal diseases, ${ }^{3,7}$ we hypothesized that smoking might show its harmful effects on periodontal tissues, in part, by influencing MMP-2 and MMP-9 expression.

Our findings revealed similar PI, PD and CAL scores for smokers and non-smokers in both $\mathrm{CP}$ and periodontally healthy group. In previous studies, ${ }^{5,24}$ non-smoker CP group has significantly higher GI scores than smoker $\mathrm{CP}$ group whereas in periodontally healthy group GI scores were similar. In an earlier study 369 periodontitis patients were evaluated and, by our results, smokers exhibited fewer gingival bleeding sites than did non-smokers. ${ }^{25}$ This issue further researched in an experimental gingivitis model that conducted in smoker and non-smoker dental students. This study showed that although dental plaque accumulation was similar in both groups, the number of gingival bleeding sites, the amount of gingival exudate and the number of gingival sites with distinct redness were less in smokers. ${ }^{26}$ Both of these two studies support the suppressed inflammatory gingival response in smokers. The mechanism by which smoking suppresses gingival bleeding is unknown, but one possible reason is the vasoconstrictor effect of nicotine. By this way, nicotine reduces blood flow, edema, and inhibits early signs of gingival inflammation. ${ }^{27,28}$

Consistent with the properties of nicotine mentioned above, smoker $\mathrm{CP}$ group revealed lower scores than non-smoker $\mathrm{CP}$ group regarding histologic parameters such as inflammation, neovascularization and fibroblastic proliferation. These findings are also supported by previous in vitro studies that demonstrate the adverse effect of nicotine on human gingival fibroblasts attachment and viability. ${ }^{29,30}$

MMPs are enzymes that can degrade extracellular matrix (ECM) and basement membrane (BM) components. These enzymes play a critical role in the degradation and remodeling of ECM and BM during active periodontitis. ${ }^{8} \mathrm{MMP}-2$ and MMP-9 that belong to gelatinases play essential roles in tissue destruction in periodontal disease. Although the exact mechanism is still unknown, gingival crevicular fluid levels of these gelatinases detected in increased levels in periodontitis patients. ${ }^{31,32}$ In another study Smith et al. compared the expression of MMP-9 in gingival tissues of periodontitis and periodontally healthy patients. Higher MMP-9 level was found in periodontitis group compared to periodontally healthy group. ${ }^{12}$ Similar to previous studies, our results indicated higher MMP-2 and MMP-9 expression in the periodontitis group compared to the periodontally healthy group. Also, MMP-2 and MMP-9 levels positively correlated with inflammation, neovascularization and fibroblastic proliferation scores. Based on our findings, we concluded that our results support the role of MMP-2 and MMP-9 in the pathogenesis of periodontitis.

The effect of smoking on gelatinases investigated in a limited number of study, and these earlier studies have yielded contradictory results on the impact of tobacco on MMPs. Neto et al. assessed the association between smoking and MMP-2 expression in experimental periodontitis model in rats. They found no association between smoking and MMP-2 expression in healthy gingiva whereas in the ligated group elevated MMP-2 expression detected in cigarette smoke inhalation group. ${ }^{14}$ In a clinical study possible relationship between smoking and serum concentration of MMP8,-9, tissue inhibitor of matrix metalloproteinases-1 (TIMP-1), neutrophil elastase (NE), and myeloperoxidase (MPO) in chronic periodontitis patients relatively to periodontally healthy subjects were investigated. Serum MMP-9 levels detected in highher levels in smoker CP group. ${ }^{13}$ In contrast, Raitao et al. conducted a study to compare the levels of MMP-2,-9 and -8 in the skin, serum and saliva of smokers and non-smokers and found lower levels of MMP-9 in saliva of smoker group compared to non-smokers whereas a tendency towards higher levels of MMP-2,-8,-9 were detected 
in the serum of smokers. It concluded that the effects of smoking on the levels of MMPs vary in different human tissues and fluids. ${ }^{33}$

In the present study, we found lower MMP-2 and MMP-9 expression in smoker CP patients compared to non-smoker $\mathrm{CP}$ group. Confirming our findings, previous studies have also reported decreased levels of MMP-2 in tissues of smokers, when compared to non-smokers with periodontitis. ${ }^{34}$ The lower MMP-2 and -9 in smokers in contrast to non-smokers with periodontitis can be explained by the fact that the reactive oxygen species present in cigarette smoke not only activate latent pro-MMPs but also inactivate fragment MMPs. ${ }^{35,36}$ Also, cigarette smoke-derived compounds such as nicotine might down-regulate the expression of MMP-9. ${ }^{37,38}$ However, in contrast to the present study, Mouzakiti et al. ${ }^{39}$ demonstrated that smokers presented higher expression of MMP-9 in comparison with non-smokers. Possible explanations for the differences mentioned above among studies may be the severity of periodontitis in the sampling site or the frequency and duration of the smoking habit. In the present study, smoking status determined by self-reports of the people. Recently, it was reported that self-reports of non-smoking people could sometimes be unreliable. ${ }^{16}$ Thus, confirmation of the smoking status by serum or salivary cotinine analyses may result in changing the groups, and this may affect the results. Therefore, determination of smoking status by only self-reports may be regarded as a limitation of this study.

In periodontally healthy group smoking did not lead a difference in MMP-2 expression whereas it increased MMP-9 expression. MMP-9 secreted from neutrophil, keratinocytes, endothelial cells, monocytes, osteoblasts, and chondrocytes whereas polymorphonuclear leukocytes do not secrete MMP$2{ }^{40,41}$ Previous studies showed that smoking might alter neutrophil behavior in periodontal tissues. ${ }^{42}$ The increased MMP-9 positivity independently from MMP 2 positivity in smokers compared to non-smokers in the healthy group may be explained by the effects of smoking on the behavior of the neutrophils.

In the present study, MMPs expression evaluated immunohistochemically according to the presence or absence of the reaction. Lack of the evaluation of mRNA expression of gelatinases and the assessment of the precursor and active forms of gelatinases is another limitation of the present study. In conclusion, our present finding of significantly increased expression of gelatinases obtained in $\mathrm{CP}$ group compared to a periodontally healthy group provides further support for the role of gelatinases in chronic periodontitis pathogenesis. The present data also suggest that smoking seems to lower the gingival tissue expression of MMP-2 and -9 in the CP group whereas it increases MMP-9 in the periodontally healthy group. The cross-sectional design of the present study presents limitations and prevents to establish the exact cause and effect relationship. Therefore, it is important to be aware of these limitations when the current results interpreted. Further studies that assess the mRNA expression of gelatinases in the gingival tissues with larger sample size required for a more detailed evaluation of the effects of smoking on gingival tissues.

\section{Acknowledgments}

This research supported by Baskent University Research Fund (D-DA11/07). The authors thank Prof. Dr. Meric Yavuz Colak, for her contributions in statistical analysis.

\section{References}

\footnotetext{
1. Bastos MF, Tucci MA, de Siqueira A, de Faveri M, Figueiredo LC, Vallim PC et al. Diabetes may affect the expression of matrix metalloproteinases and their inhibitors more than smoking in chronic periodontitis.

J Periodontal Res. 2017 Apr;52(2):292-9.

https://doi.org/10.1111/jre.12394
}

2. Genco RJ, Borgnakke WS. Risk factors for periodontal disease. Periodontol 2000. 2013 Jun;62(1):59-94. https://doi.org/10.1111/i.1600-0757.2012.00457.x

3. Johnson GK, Guthmiller JM. The impact of cigarette smoking on periodontal disease and treatment. Periodontol 2000. 2007;44(1):17894. https://doi.org/10.1111/j.1600-0757.2007.00212.x 
4. Trombelli L, Kim CK, Zimmerman GJ, Wikesjö UM. Retrospective analysis of factors related to clinical outcome of guided tissue regeneration procedures in intrabony defects. J Clin Periodontol. 1997 Jun;24(6):366-71. https://doi.org/10.1111/j.1600-051X.1997.tb00199.x

5. Preber $\mathrm{H}$, Bergström J. Effect of cigarette smoking on periodontal healing following surgical therapy. J Clin Periodontol. 1990 May;17(5):324-8. https://doi.org/10.1111/j.1600-051X.1990.tb01098.x

6. Kinane DF, Radvar M. The effect of smoking on mechanical and antimicrobial periodontal therapy. J Periodontol. 1997 May;68(5):467-72. https://doi.org/10.1902/jop.1997.68.5.467

7. Nociti FH Jr, Casati MZ, Duarte PM. Current perspective of the impact of smoking on the progression and treatment of periodontitis. Periodontol 2000. 2015 Feb;67(1):187-210. https://doi.org/10.1111/prd.12063

8. Sorsa T, Tjäderhane L, Salo T. Matrix metalloproteinases (MMPs) in oral diseases. Oral Dis. 2004 Nov;10(6):311-8. https://doi.org/10.1111/j.1601-0825.2004.01038.x

9. Johannsen A, Susin C, Gustafsson A. Smoking and inflammation: evidence for a synergistic role in chronic disease. Periodontol 2000. 2014 Feb;64(1):111-26. https://doi.org/10.1111/j.1600-0757.2012.00456.x

10. Boström L, Linder LE, Bergström J. Smoking and crevicular fluid levels of IL- 6 and TNF-alpha in periodontal disease. J Clin Periodontol. 1999 Jun;26(6):352-7. https://doi.org/10.1034/j.1600-051X.1999.260604.x

11. Söder B, Jin LJ, Wickholm S. Granulocyte elastase, matrix metalloproteinase- 8 and prostaglandin E2 in gingival crevicular fluid in matched clinical sites in smokers and non-smokers with persistent periodontitis. J Clin Periodontol. 2002 May;29(5):38491. https://doi.org/10.1034/i.1600-051X.2002.290502.x

12. Smith PC, Muñoz VC, Collados L, Oyarzún AD. In situ detection of matrix metalloproteinase-9 (MMP9) in gingival epithelium in human periodontal disease. J Periodontal Res. 2004 Apr;39(2):87-92. https://doi.org/10.1111/j.1600-0765.2004.00705.x

13. Ozçaka O, Biçakci N, Pussinen P, Sorsa T, Köse T, Buduneli N. Smoking and matrix metalloproteinases, neutrophil elastase and myeloperoxidase in chronic periodontitis. Oral Dis. 2011 Jan;17(1):68-76. https://doi.org/10.1111/j.1601-0825.2010.01705.x

14. César Neto JB, Souza AP, Barbieri D, Moreno Junior H, Sallum EA, Nociti Junior FH. Matrix metalloproteinase-2 may be involved with increased bone loss associated with experimental periodontitis and smoking: a study in rats. J Periodontol. 2004 Jul;75(7):995-1000. https://doi.org/10.1902/jop.2004.75.7.995

15. Elm E, Altman DG, Egger M, Pocock SJ, Gøtzsche PC, Vandenbroucke JP. The Strengthening the Reporting of Observational Studies in Epidemiology (STROBE) statement: guidelines for reporting observational studies. Int J Surg. 2014 Dec;12(12):1495-9. https://doi.org/10.1016/j.ijsu.2014.07.013

16. Buduneli N, Kardeşler L, Işik H, Willis CS 3rd, Hawkins SI, Kinane DF et al. Effects of smoking and gingival inflammation on salivary antioxidant capacity. J Clin Periodontol. 2006 Mar;33(3):159-64. https://doi.org/10.1111/j.1600-051X.2006.00892.x

17. Armitage GC. Development of a classification system for periodontal diseases and conditions. Ann Periodontol. 1999 Dec;4(1):1-6. https://doi.org/10.1902/annals.1999.4.1.1

18. Loe $\mathrm{H}$, Silness J. Periodontal disease in pregnancy. I. Prevalence and severity. Acta Odontol Scand. 1963 Dec;21(6):533-51. https://doi.org/10.3109/00016356309011240

19. Noack B, Kipping T, Tervahartiala T, Sorsa T, Hoffmann T, Lorenz K. Association between serum and oral matrix metalloproteinase- 8 levels and periodontal health status. J Periodontal Res. 2017 Oct;52(5):824-31. https://doi.org/10.1111/ire.12450

20. Silness J, Loe H. Periodontal disease in pregnancy. II. Correlation between oral hygiene and periodontal condtion. Acta Odontol Scand. 1964 Feb;22(1):121-35. https://doi.org/10.3109/00016356408993968

21. Moimaz SA, Zina LG, Saliba O, Garbin CA. Smoking and periodontal disease: clinical evidence for an association. Oral Health Prev Dent. 2009;7(4):369-76.

22. Bergström J. Tobacco smoking and risk for periodontal disease. J Clin Periodontol. 2003 Feb;30(2):107-13. https://doi.org/10.1034/i.1600-051X.2003.00272.x

23. Sullivan KM, Dean A, Soe MM. OpenEpi: a webbased epidemiologic and statistical calculator for public health. Public Health Rep. 2009 May-Jun;124(3):471-4. https://doi.org/10.1177/003335490912400320

24. Rivera-Hidalgo F. Smoking and periodontal disease. Periodontol 2000. 2003;32(1):50-8. https://doi.org/10.1046/i.0906-6713.2003.03205.x

25. Preber $\mathrm{H}$, Bergström J. Cigarette smoking in patients referred for periodontal treatment. Scand J Dent Res. 1986 Apr;94(2):102-8.

26. Bergström J, Preber H. The influence of cigarette smoking on the development of experimental gingivitis. J Periodontal Res. 1986 Nov;21(6):668-76. https://doi.org/10.1111/j.1600-0765.1986.tb01504.x

27. Dietrich T, Bernimoulin JP, Glynn RJ. The effect of cigarette smoking on gingival bleeding. J Periodontol. 2004 Jan;75(1):16-22. https://doi.org/10.1902/jop.2004.75.1.16

28. Turnbull B. Smoking and periodontal disease: a review. J N Z Soc Periodontol. 1995;(79):10-5.

29. Esfahrood ZR, Zamanian A, Torshabi M, Abrishami M. The effect of nicotine and cotinine on human gingival fibroblasts attachment to root surfaces. J Basic Clin Physiol Pharmacol. 2015 Sep;26(5):517-22. https://doi.org/10.1515/ibcpp-2014-0120

30. Austin GW, Cuenin MF, Hokett SD, Peacock ME, Sutherland $\mathrm{DE}$, Erbland JF et al. Effect of nicotine on fibroblast beta 1 integrin expression and distribution in vitro. J Periodontol. 2001 Apr;72(4):438-44. https://doi.org/10.1902/jop.2001.72.4.438

31. Ejeil AL, Igondjo-Tchen S, Ghomrasseni S, Pellat B, Godeau G, Gogly B. Expression of matrix metalloproteinases (MMPs) and tissue inhibitors of metalloproteinases (TIMPs) in healthy and diseased human gingiva. J Periodontol. 2003 Feb;74(2):18895. https://doi.org/10.1902/jop.2003.74.2.188 
32. Pozo P, Valenzuela MA, Melej C, Zaldívar M, Puente J, Martínez $\mathrm{B}$ et al. Longitudinal analysis of metalloproteinases, tissue inhibitors of metalloproteinases and clinical parameters in gingival crevicular fluid from periodontitis-affected patients. J Periodontal Res. 2005 Jun;40(3):199-207. https://doi.org/10.1111/j.1600-0765.2005.00786.x

33. Raitio A, Tuomas H, Kokkonen N, Salo T, Sorsa T, Hanemaaijer R et al. Levels of matrix metalloproteinase-2, -9 and -8 in the skin, serum and saliva of smokers and non-smokers. Arch Dermatol Res. 2005 Dec;297(6):242-8. https://doi.org/10.1007/s00403-005-0597-1

34. César-Neto JB, Duarte PM, Oliveira MC, Tambeli CH, Sallum EA, Nociti Junior FH. Smoking modulates interleukin6:interleukin-10 and RANKL:osteoprotegerin ratios in the periodontal tissues. J Periodontal Res. 2007 Apr;42(2):18491. https://doi.org/10.1111/j.1600-0765.2006.00934.x

35. Liede KE, Haukka JK, Hietanen JH, Mattila MH, Rönkä $H$, Sorsa T. The association between smoking cessation and periodontal status and salivary proteinase levels. J Periodontol. 1999 Nov;70(11):1361-8. https://doi.org/10.1902/jop.1999.70.11.1361

36. Saari H, Suomalainen K, Lindy O, Konttinen YT, Sorsa T. Activation of latent human neutrophil collagenase by reactive oxygen species and serine proteases. Biochem Biophys Res Commun. 1990 Sep;171(3):979-87. https://doi.org/10.1016/0006-291X(90)90780-Q
37. Renò F, Rocchetti V, Migliario M, Cannas M. Nicotine modulates gelatinase B (MMP-9) and epilysin (MMP-28) expression in reconstituted human oral epithelium. J Oral Pathol Med. 2011 Jan;40(1):33-6. https://doi.org/10.1111/j.1600-0714.2010.00949.x

38. Hatta T, Sano H, Sakamoto N, Kishimoto KN, Sato M, Itoi E. Nicotine reduced MMP-9 expression in the primary porcine tenocytes exposed to cyclic stretch. J Orthop Res. 2013 Apr;31(4):645-50. https://doi.org/10.1002/jor.22259

39. Mouzakiti E, Pepelassi E, Fanourakis G, Markopoulou C, TseleniBalafouta $S, V$ rotsos I. The effect of smoking on the mRNA expression of MMPs and TIMP-1 in untreated chronic periodontitis patients: a cross-sectional study. J Periodontal Res. 2011 Oct;46(5):576-83. https://doi.org/10.1111/j.1600-0765.2011.01375.x

40. Weiss SJ. Tissue destruction by neutrophils. N Engl J Med. 1989 Feb;320(6):365-76. https://doi.org/10.1056/NEJM198902093200606

41. Zucker S, Lysik RM, Gurfinkel M, Zarrabi MH, Stetler-Stevenson W, Liotta LA et al. Immunoassay of type IV collagenase/gelatinase (MMP-2) in human plasma. J Immunol Methods. 1992 Apr;148(12):189-98. https://doi.org/10.1016/0022-1759(92)90172-P

42. Liu KZ, Hynes A, Man A, Alsagheer A, Singer DL, Scott DA. Increased local matrix metalloproteinase- 8 expression in the periodontal connective tissues of smokers with periodontal disease. Biochim Biophys Acta. 2006 Aug;1762(8):775-80. https://doi.org/10.1016/j.bbadis.2006.05.014 